\title{
Local Adaptive Automatic Binarisation (LAAB)
}

\author{
T.Romen Singh \\ Department of I.T, School of \\ Technology, Assam University, \\ Silchar - 788011, Assam, India
}

\author{
Sudipta Roy \\ Department of I.T, School of \\ Technology, Assam University, \\ Silchar - 788011, Assam, India
}

\author{
Kh. Manglem Singh \\ Department of Computer Science \\ and Engineering, NIT, Manipur \\ Imphal -795001, Manipur, India.
}

\begin{abstract}
Most of the binarization techniques associate a certain intensity value called threshold which separate the pixel values of the concerned input grey scale image into two classes like background and foreground. Each and every pixel should be compared with the threshold and transformed to its respective class according to the threshold value. In this paper an automatic binarisation technique with local adaptation without any intensity value (threshold) of partition, is described. It creates a binarised image by transforming the input image to its respective binarised image automatically without using any threshold value. It uses local mean to adapt to local environment within a window of size $w \times w$. Local mean determination is time consuming one and to reduce the time consumption, integral sum image is used as prior process. The input grey scale image is self transformed to an integral sum image within itself and then transform to binary image from the integral sum image itself.
\end{abstract}

\section{Keywords}

Automatic, Binarisation, local adaptive, integral sum image, autobinarization.

\section{INTRODUCTION}

Image binarization plays a key role in the field of Image Processing. In general, binarization is a process of transforming a grey scale image to a binary image which contains only two classes of pixels white as background and black as foreground. Classification is carried out with a certain separation intensity value called threshold. Threshold plays a major role in binarisation and choosing of an appropriate threshold value is an important one. There are many techniques of determining an appropriate threshold value in binarisation techniques. Binarisation can be done in two ways as global and local thresholding techniques.

In the global technique, a single threshold is selected for the entire input image and each pixel is compared with the selected threshold value at the time of transformation. Global thresholding [2] is an efficient, less time consuming and region independent one. It is more appropriate in the uniformly illuminated images than the badly illuminated images like document images.

In local adaptive techniques [3-22], a threshold value is determined for each pixel depending on the neighboring pixels within a local window. Proper choice of threshold value leads to the high quality of binary image. Determination of local threshold value of each pixel within a local window is time consuming. But the local technique can solve the problem of binarisation of badly illuminated images. To reduce the time consumption some local technique [22 ] use integral sum image[1] as prior process.
In this technique of binarisation, no threshold value is determined and instead, it transform the input image to a binary image by adapting the local region within a window automatically. Due to the local adaptation, it is also time consuming one like other local techniques. To reduce the time consumption, it use integral sum image[1] transformation as a prior process. The difference from other techniques of transforming to integral sum image is that here the transformation is within the input image itself. It can save the space occupied by the input image. Other techniques use three image files such as input image, integral sum image and binarised image file simultaneously.

\section{PROPOSED ALGORITHM}

Local thresholding techniques determine a threshold $t(x, y)$ within a local window of size $w \times w$ such that

$$
b(x, y)=\left\{\begin{array}{lcc}
0 & \text { if } & g(x, y) \leq t(x, y) \\
1 & & \text { otherwise }
\end{array}\right.
$$

where $b(x, y) \in\{0,1\}$ is the binarised image and $g(x, y) \in[0,1]$ is the input grey scale image.

In this technique of binarisation LAAB, the input image $g(x, y)$ is transformed to a binary image $b(x, y)$ automatically without using any threshold $t(x, y)$ by adapting a local region of size $w \times w$. Adaptation is carried out with the help of the local mean $m(x, y)$. Local mean calculation is time consuming depending on the local region size $w$. If integral sum is used to determine local mean, its computational time is independent of local region size $w$. This technique of binarisation LAAB input image itself is transformed to integral sum image as a prior process and then transformed to a binary image from the integral sum image itself. Self integral image representation save space to be occupied by input image during binarisation.

\subsection{Self Transformed Integral Sum Image} Integral sum image $g$ of a gray scale image $\boldsymbol{I}$ is defined as

$$
g(x, y)=\sum_{i=1}^{x} \sum_{j=1}^{y} I(i, j)
$$

Transformation can be done within the input image itself instead of transforming to another image. Transformation of 
an input image $g$ to an integral sum image within itself is carried out with the following steps:

First raw : $g(1, y)=g(1, y-1)+g(1, y), y=2 . . n$

First column : $g(x, 1)=g(x-1,1)+g(x, 1), x=2 . . m(4)$

Other points at $(x, y)$ :

$g(x, y)=g(x, y)+g(x-1, y)+g(x, y-1)-g(x-1, y-1)$

$x=2 . . m, \quad y=2 . . n$

\begin{tabular}{|c|c|c|c|c|c|c|c|c|c|}
\hline & \multicolumn{7}{|c|}{$g(x, y)$ Integral sum } \\
\hline \multirow{2}{*}{\multicolumn{2}{|c|}{\begin{tabular}{l|l|l|l|}
1 & 1 & 2 & 1 \\
\end{tabular}}} & & \multirow{2}{*}{$1 / 4$} & \multirow[b]{2}{*}{$\begin{array}{ll}1 & 5 \\
& \end{array}$} & \multirow[b]{2}{*}{1} & \multirow[b]{2}{*}{1} & \multirow[b]{2}{*}{1} & \multirow[b]{2}{*}{1} & \multirow[b]{2}{*}{1} \\
\hline & & & & & & & & & \\
\hline $\begin{array}{|ll|}1 & 2 \\
\end{array}$ & 1 (4) & & ) 8 & 110 & 1 & 1 & 1 & 1 & 1 \\
\hline \begin{tabular}{|l|l|}
1 & 3 \\
\end{tabular} & 16 & (1) (9) & & 1 & 1 & 1 & 1 & 1 & 1 \\
\hline $\begin{array}{|ll|}1 & 4 \\
& 4\end{array}$ & 18 & 1 & 1 & 1 & 1 & 1 & 1 & 1 & 1 \\
\hline $\begin{array}{|ll|}1 & 5 \\
& 5\end{array}$ & 110 & 1 & 1 & 1 & 1 & 1 & 1 & 1 & 1 \\
\hline \multicolumn{5}{|c|}{$\uparrow$} & \multicolumn{5}{|c|}{$\downarrow$} \\
\hline $\begin{array}{|ll|}1 & 1 \\
& 1 \\
\end{array}$ & 12 & & 14 & $4^{1} 5$ & 1 & 12 & 13 & \begin{tabular}{ll|}
1 & 4 \\
\end{tabular} & 15 \\
\hline $\begin{array}{|ll|}1 & 2 \\
& 2 \\
\end{array}$ & 1 & 1 & 1 & 1 & 1 & 1 & 1 & 1 & 1 \\
\hline $\begin{array}{|ll|}1 & 3 \\
& 3\end{array}$ & 1 & 1 & 1 & 1 & 1 & 1 & 1 & 1 & 1 \\
\hline $\begin{array}{|ll|}1 & 4 \\
& 4\end{array}$ & 1 & 1 & 1 & 1 & 1 & 1 & 1 & 1 & 1 \\
\hline $\begin{array}{|ll|}1 & 5 \\
& 5\end{array}$ & 1 & 1 & 1 & 1 & 1 & 1 & 1 & 1 & 1 \\
\hline
\end{tabular}

Fig 1: Integral sum image self transformation sequence

Using the above three equations, Eqn(3-5) a gray scale input image $g$ can be transformed to an integral sum image within itself. Its diagrammatic representation is shown in Figure 1. The dimmed numbers indicate the original pixel values where the dark numbers indicate the calculated integral sum value at their respective positions. Circled numbers indicate the current concerned pixel values used to determine integral sum image $g(x, y)$ at $(x, y)$.

Once we have an integral sum image $g$, we can determine the local sum $s(x, y)$ of pixels at $(x, y)$ within a window of size $w \times w$ using $\operatorname{Eqn}(7)$. The original pixel value of $g(x, y)$ at $(x, y)$ also can be determined using Eqn(6). From the local sum, we can determine the local mean $m(x, y)$ using $\operatorname{Eqn}(8)$.

$$
\begin{aligned}
& g(x, y)=g(x, y)-[g(x, y-1)+g(x-1, y)]+g(x-1, y-1) \\
& s(x, y)=g(x+d, y+d)+(x-d-1, y-d-1) \\
& -[g(x-d, y-d-1)+g(x-d-1, y-d)]
\end{aligned}
$$

where $d=\frac{w+1}{2}, w$ is the length of local window and $(x, y)$ is at the centre of the window.

$$
m(x, y)=\frac{s(x, y)}{w^{2}}
$$

\subsection{Proposed Technique (LAAB)}

Automatic binarisation is a process of transforming a gray scale image $g(x, y)$ to a binary image $b(x, y)$ automatically without using any threshold $t(x, y)$ by adapting the pixels within a local region environment. This paper describe an automatic binarisation with local adaptation. Local adaptation is carried out within a local window of size $w \times w$ with the help of local mean $m(x, y)$ of pixel intensity values of pixels within the local region.

The automatic binarisation is designed as:

$b(x, y)=\frac{|1-2 v|-(1-2 v)}{2|1-2 v|}$

where $\quad v=\frac{k(1+\partial)}{(1-\partial)}, \quad k$ is a bias such that

$0.5<k<0.6, \partial=\{g(x, y)-m(x, y)\}\{1-m(x, y)\}$,

$g(x, y)$ is the original pixel at $(x, y)$ given by $\operatorname{Eqn}(6)$.

Since $0 \leq g(x, y) \leq 1$ and $0 \leq m(x, y) \leq 1$, we get $-1<\partial<1$ and $1-\partial>0$

The bias $k$ control the level of adaptation of pixels within the local region at the time of transformation to the binarised image $b(x, y)$. The greater the value of $k$, the more area of background and less area of foreground and vice versa. .

\section{EXPERIMENTAL RESULT}

The proposed technique (LAAB) is tested on many categories of images like medical image, Scanned document images, Camera captured document images and non document images. Figures 3-5 show some of the tested image. The experiment was carried out using MATLAB 7.3 (R2006b) on a PC with the following configuration: Intel ${ }^{\circledR}$ Core ${ }^{\mathrm{TM}} 2 \mathrm{Duo} \mathrm{CPU}$ E6550, GHz $2.33 \mathrm{GHz}, 2 \mathrm{~GB}$ RAM, 32 bit OS(Windows Vista). Figures 3-5 show the test results comparing with other techniques. Figure 2 shows the graphical time complexity comparison of table 1 which shows the comparison of the LAAB with other local techniques. From the experimental result we find that $\mathrm{LAAB}$ gives similar result to other techniques like Sauvola[9] and T.R. Singh [22] for some type of images as in Figure 3 and 5. But some type of images as in Figure 4 , LAAB give better result than the other techniques.

Due to uses of integral sum image, the computational time is independent of local window size $w$ like T.R Singh while other techniques depend on local window size $w$. Hence its 
time complexity is $O\left(n^{2}\right)$ while the region size $(w)$ dependent techniques have $O\left(n^{2} \times w^{2}\right)$. Its computational time comparison is shown in Figure 2 and Table 1. Based on the experimental results LAAB is better than the other techniques.

Table 1 : Computational Time Comparison of document image $_{480 \times 188}$ which is shown in figure 2 .

\begin{tabular}{|l|r|r|r|r|r|}
\hline $\begin{array}{c}\text { Size } \\
\text { of } \boldsymbol{W}\end{array}$ & $\begin{array}{c}\text { T.R. } \\
\text { Singh }\end{array}$ & Bernsen & Niblack & Sauvola & LAAB \\
\hline 3 & 0.0624 & 0.4212 & 4.4304 & 4.4304 & 0.0624 \\
\hline 9 & 0.0624 & 0.9984 & 4.4616 & 4.446 & 0.0624 \\
\hline 15 & 0.0468 & 1.9032 & 4.8828 & 4.8672 & 0.078 \\
\hline 21 & 0.0624 & 3.0888 & 5.4912 & 5.3976 & 0.0624 \\
\hline 27 & 0.0468 & 4.524 & 6.4272 & 6.1776 & 0.0468 \\
\hline
\end{tabular}

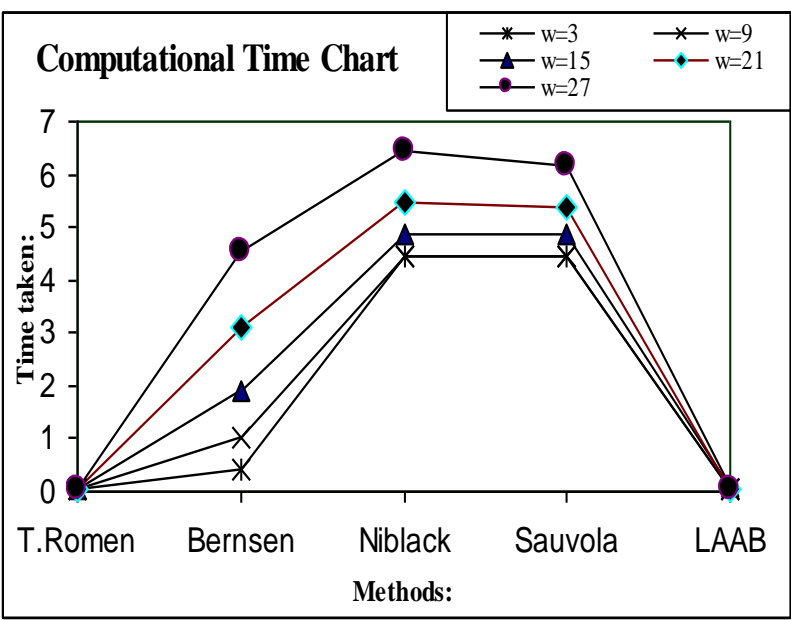

Fig 2: Computational time chart at different window size.

\section{CONCLUSION}

This paper (LAAB) describes a new way of local automatic binarisation technique without using threshold value. Its computational time complexity is $O\left(n^{2}\right)$ which is very close to the global techniques, while other local techniques like Sauvola, Bernsen, and Niblack have $O\left(n^{2} \times w^{2}\right)$ for an image of size $n \times n$. To reduce computational time complexity to $O\left(n^{2}\right)$ LAAB use integral sum image like T.R.Singh[22] as a prior process. Hence the experimental result is compared with other local techniques including T.R. Singh. Using of integral sum image representation face a problem of representing a large sum value at a particular point. Further it can be improve with a suitable integral sum image presentation technique which is free from large sum value representation problem.

\section{ACKNOWLEDGMENTS}

We thank to all the authors who enlighten our knowledge towards development of this paper for publication. We also thank O. Imocha Singh, Head, Department of Computer Science, Manipur University for his valuable technical support to develop this paper successfully.
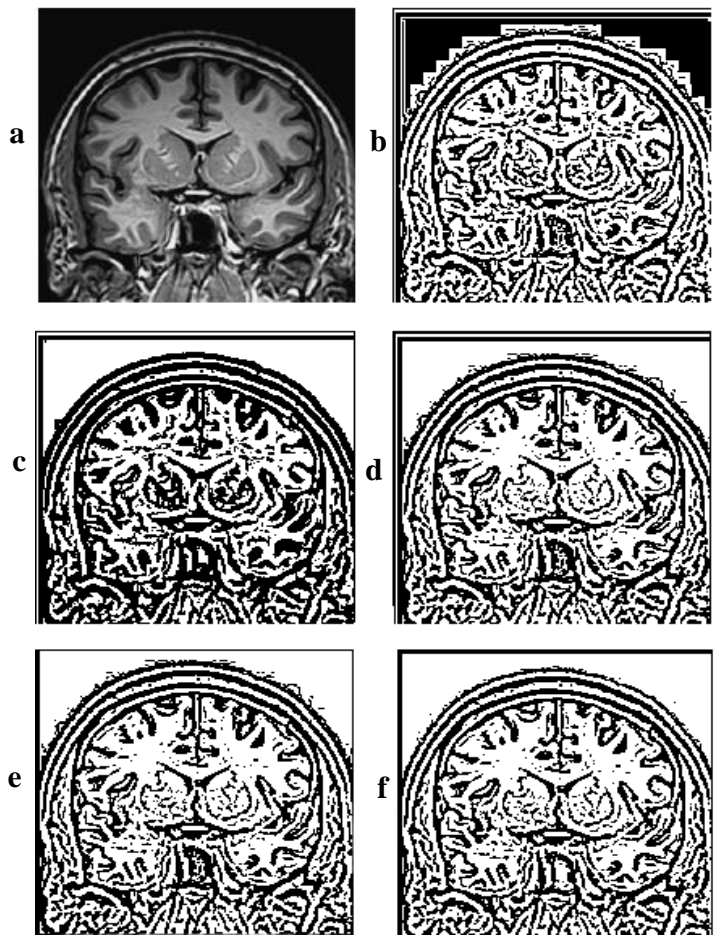

Fig 3: Binarised result of various method on document image at $15 \times 15$ window size: a. original image $b$. Niblack

at $k=-0.25$ c. Bernsen's d. Sauvola's at $k=0.1$

e. T.R Singh's at $k=0.1$ and $f$. LAAB at $k=0.51$
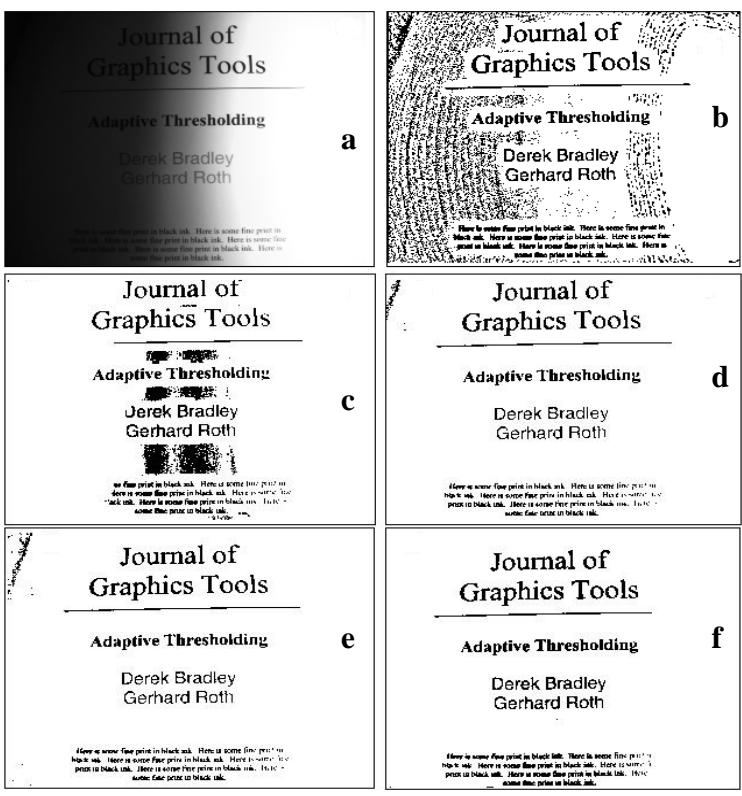

Fig 4: Binarised result of various method on document image at $15 \times 15$ window size: a. original image $b$. Niblack at $\mathrm{k}=-\mathbf{0 . 2 5} \mathrm{c}$. Bernsen's d. Sauvola's at $k=0.2$ e. T.R. Singh's at $k=0.2$ and $f$. $\mathrm{LAAB}$ at $\mathrm{k}=\mathbf{0 . 5 3}$ 


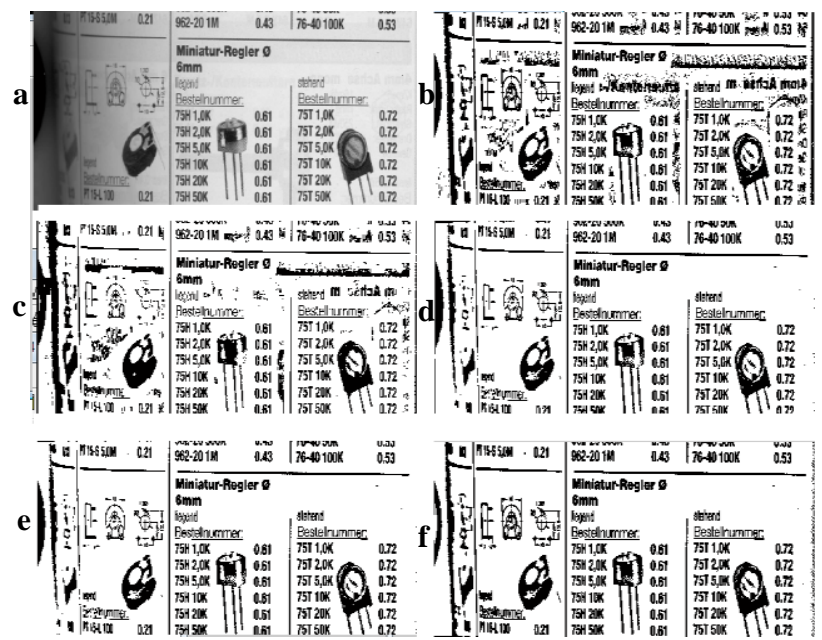

Fig 5: Binarised result of various method on document image at $15 \times 15$ window size: a. original image $b$. Niblack at $\mathrm{k}=\mathbf{- 0 . 2 5}$ c. Bernsen's d. Sauvola's at $\mathrm{k}=\mathbf{0 . 1 5}$ e. T.R. Singh's at $k=0.15$ and $f$. LAAB at $k=0.51$

\section{REFERENCES}

[1] Konstantinos G. Derpanis," Integral image-based representations", Viola, P. \& Jones, M. (2001). Rapid object detection using a boosted cascade of simple features. In IEEE Computer Vision and Pattern Recognition (pp. I:511-518).

[2] N. Otsu, 1979 A threshold selection method from graylevel histograms, IEEE Trans. Systems, Man, and Cybernetics 9(1), pp.62-66.

[3] Bernsen, J. 1986, Dynamic thresholding of gray-level images. Proc. $8^{\text {th }}$ Int. Conf. on Pattern Recognition, Paris, , pp. 1251-1255

[4] W. Niblack, 1986 An Introduction to Image Processing, Prentice-Hall, Englewood Cliffs, NJ,

[5] Gorman, L.O. 1994, Binarization and multithresholding of document images using connectivity', CVGIP, Graph. Models Image Process., 56, (6), pp. 494-506

[6] O.D. Trier and A. K. Jain, 1995. "Goal-directed evaluation of binarization methods," IEEE Trans. Pattern Anal. Mach. Intell. PAMI-17, 1191- 1201

[7] Sauvola, J., Seppanen, T., Haapakoski, S., and Pietikainen, M.: 'Adaptive document binarization'. Proc. 4th Int. Conf. on Document Analysis and Recognition, Ulm Germany, 1997, pp. 147-152

[8] Liu, Y., and Srihari, S.N.: 'Document image binarization based on texture features', IEEE Pattern Anal. Mach. Intell., 1997, 19, (5), pp. 540-544

[9] J. Sauvola and M. Pietikainen, "Adaptive document image binarization," Pattern Recognition 33(2), pp. 225236, 2000.

[10] M. Sezgin and B. Sankur, "Comparison of thresholding methods for non-destructive testing applications," IEEE ICIP'2001, Intl. Conf. Image Process., pp. 764-767 (2001).
[11] P. Viola and M. J. Jones, 2004 "Robust real-time face detection," Int. Journal of Computer Vision 57(2), pp. $137-154$,

[12] Mehmet Sezgin and Bu" lent Sankur, 2004 "Survey over image thresholding techniques and quantitative performance evaluation", Journal of Electronic Imaging 13(1), 146-165 (January).

[13] F. Shafait, D. Keysers, and T. M. Breuel, "Performance comparison of six algorithms for page segmentation," in 7th IAPR Workshop on Document Analysis Systems, pp. 368-379, (Nelson, New Zealand), Feb. 2006.

[14] B. Gatos, I. Pratikakis and S.J. Perantonis,' Improved Document Image Binarization by Using a Combination of Multiple Binarization Techniques and Adapted Edge Information', 978-1-4244-2175-6/08/\$25.00 (C2008 IEEE

[15] B. Su, S. Lu, and C. L. Tan, "Document Image Binarization Using Background Estimation and Stroke Edges," Proc. Intl. Journal on Document Analysis \&Recognition, Vol. 13, No. 4, pp. 303-314, 2010.

[16] Ioannis Pratikakis, Basilis Gatos and Konstantinos Ntirogiannis, "H-DIBCO 2010 -Handwritten Document Image Binarization Competition", 2010 12th International Conference on Frontiers in Handwriting Recognition. 978-0-7695-4221-8/10 \$26.00 (C) 2010 IEEEDOI 10.1109/ICFHR.2010.118.

[17] I. Ben Messaoud, H. Amiri, H. El Abed, and V.Märgner, "New binarization approach based on text blockextraction", in International Conference on Document Analysis and Recognition (ICDAR), 2011.

[18] T.Obafemi-Ajayi and G. Agam. "Statistical multiresolution schemes for historical document binarization". In Document Recognition and Retrieval XVIII, Proc. SPIE,2011.

[19] F. Kleber, M. Diem and R. Sablatnig. "Scale Space Binarization Using Edge Information Weighted by a Foreground Estimation", ICDAR 2011.

[20] MA. Ramírez-Ortegón, E. Dueñez-Guzmán, R. Rojas, and E. Cuevas, "Unsupervised Evaluation Measures for Binarization", Pattern Recognition, Vol. 44, issue 3, pp 491-502, 2011.

[21] Ioannis Pratikakis, Basilis Gatos and Konstantinos Ntirogiannis,"ICDAR 2011 Document Image Binarization Contest (DIBCO 2011)", 2011 International Conference on Document Analysis and Recognition, $1520-5363 / 11 \quad \$ 26.00 \quad$ (c) $2011 \quad$ IEEE DOI 10.1109/ICDAR.2011.299,pp 1506-1510.

[22] T.Romen Singh, Sudipta Roy, O.Imocha Singh, Tejmani Sinam and Kh.Manglem Singh," A New local Adaptive Thresholding Technique in Binarisation", IJCSI-Vol 8, issue 6 No. 2 pp. 271-277 (Nov, 2011).

[23] Yudong ZHANG , Lenan WU†," Fast Document Image Binarization Based on an Improved Adaptive Otsu's Method and Destination Word Accumulation", Journal of Computational Information Systems 7: 6 (2011) 18861892, 1553-9105/ Copyright @ 2011. 